JOURNAL OF

FUNCTION SPACES AND APPLICATIONS

Volume 6, Number 3 (2008), 259-276 (c) 2008, Scientific Horizon

http://www.jfsa.net

\title{
On the trace space of a Sobolev space with a radial weight
}

\section{Helmut Abels, Miroslav Krbec and Katrin Schumacher}

(Communicated by Fernando Cobos)

2000 Mathematics Subject Classification. Primary 46E35; Secondary 46E30. Keywords and phrases. weighted Sobolev spaces, Muckenhoupt weights, trace spaces.

Abstract. Our concern in this paper lies with trace spaces for weighted Sobolev spaces, when the weight is a power of the distance to a point at the boundary. For a large range of powers we give a full description of the trace space.

\section{Introduction}

We consider integer order weighted Sobolev spaces with weights equal to a power of the distance to a point of the boundary and more general weights modelled upon such weights. Our concern in this paper lies with a characterization of trace spaces of these weighted Sobolev spaces. Rather surprisingly there are not too many trace theorems for weighted Sobolev spaces even though traces belong to the fundamental concepts both in the theory and applications, and they have been studied for a very long time. One of the major reasons is that there are no straightforward analogs of methods known from the non-weighted theory, which allow a description of values on manifolds of lower dimensions. Note in passing that the study 
of traces has been closely connected with extension of integer order spaces to spaces with non-integer derivatives, and it was one of the motivation for establishing the general theory of Besov spaces.

The non-weighted theory for the $W_{p}^{k}$ was studied in many papers and it can be found in a number of well-known monographs. We shall make no attempt to make an account of that; let us collect just some of the important references. The pioneering works by Aronszajn [4] and Slobodetskii [26] for the Hilbert case and the papers by Gagliardo [12] and Stein [27] should be mentioned. The theory for $p=2$ based on abstract methods can be found in Lions and Magenes' monograph [19]. The case of general $p$ is treated for instance in monographs by Nečas [21], Adams [2], Kufner, John and Fučík [17], Bergh and Löfström [5], Triebel [29]. An immense work has been done by the Soviet school (Lizorkin, Besov, Nikol'skii, Il'in, Uspenskii, and many others). We refer to [29] for a large list of references.

Spaces with weights which equal to a power of the distance to the boundary appeared in many papers; let us refer at least to [15] and [16]. A standard approach consists in taking the trace space as a factor space (modulo equality on the boundary). Nikol'skii in his monograph [22] (especially its second edition) established a trace theorem for these Sobolev weighted spaces: For a suitable range of parameters and under assumption on the regularity on $\partial \Omega$, the boundary of $\Omega$, he identified the trace space with an unweighted Besov space with a modified smoothness parameterthe effect of the weight on the domain (Hardy's inequality behind the scenes).

Let us recall the very basic setting of the trace problem. For simplicity we shall consider spaces on $\mathbb{R}^{n}$ and traces on $\mathbb{R}^{n-1}$, that is, on $\partial \mathbb{R}_{+}^{n}$, the boundary of $\mathbb{R}_{+}^{n}=\left\{x=\left(x_{1}, \ldots, x_{n}\right) \in \mathbb{R}^{n}: x_{n}>0\right\}$. By virtue of extension theorems the Sobolev space on $\mathbb{R}_{+}^{n}$ equals (up to equivalence of norms) to the restriction of the corresponding Sobolev space on $\mathbb{R}^{n}$, equipped with the factornorm (modulo equality on $\mathbb{R}_{+}^{n}$ ). This can be transferred to spaces on a smooth domain $\Omega$ and its boundary $\partial \Omega$ in a standard way - using resolution of unity and local coordinates. Let $s>0$ be a non-integer, denote by $[s]$ the integer part of $s$, and let $1<p \leq \infty$. Then the Sobolev-Slobodetskii space $W_{p}^{s}=W_{p}^{s}\left(\mathbb{R}^{n}\right)$ is defined as the linear space of all functions $f \in L_{p}\left(\mathbb{R}^{n}\right)$ with

$$
\begin{aligned}
\left\|f \mid W_{p}^{s}\left(\mathbb{R}^{n}\right)\right\|= & \|f\|_{L_{p}\left(\mathbb{R}^{n}\right)} \\
& +\sum_{|\alpha|=[s]}\left(\int_{\mathbb{R}^{n}} \int_{\mathbb{R}^{n}} \frac{\left|D^{\alpha} f(x)-D^{\alpha} f(y)\right|^{p}}{|x-y|^{(s-[s]) p+N}} d x d y\right)^{1 / p}<\infty .
\end{aligned}
$$


(Note that this is a special case of a general Besov space $B_{p, q}^{s}\left(\mathbb{R}^{n}\right)$ for $p=q$.) Here and in the following we shall use the notation $\|f \mid X\|$ instead of $\|f\|_{X}$ whenever it might improve legibility of the text. Recall that $C\left(\overline{\mathbb{R}_{+}^{n}}\right) \cap W_{p}^{1}\left(\mathbb{R}_{+}^{n}\right)$ is dense in $W_{p}^{1}\left(\mathbb{R}_{+}^{n}\right)$. One can prove that there is a bounded linear operator

$$
\operatorname{tr}: W_{p}^{1}\left(\mathbb{R}^{n}\right) \rightarrow W_{p}^{1-1 / p}\left(\partial \mathbb{R}_{+}^{n}\right)
$$

such that $\operatorname{tr} f\left(y^{\prime}\right)=f\left(y^{\prime}\right)$ for every $y^{\prime} \in \partial \mathbb{R}_{+}^{n}$ and every $f \in C\left(\overline{\mathbb{R}_{+}^{n}}\right)$. This gives a natural meaning to values of a general $f \in W_{p}^{1}\left(\mathbb{R}_{+}^{n}\right)$ on $\partial \mathbb{R}_{+}^{n}$. Moreover, it is well known that there exists a bounded linear operator

$$
\text { ext }: W_{p}^{1-1 / p}\left(\partial \mathbb{R}_{+}^{n}\right) \rightarrow W_{p}^{1}\left(\mathbb{R}_{+}^{n}\right)
$$

such that extotr $=$ id on $W_{p}^{1-1 / p}\left(\partial \mathbb{R}_{+}^{n}\right)$. Theorems of this kind are now well-known in a general setting of Besov and Lizorkin-Triebel spaces; we refer to [29].

Now let $w$ be a weight function (shortly a weight) in $\mathbb{R}^{n}$, that is, $w \in L_{1, \text { loc }}$ and $w>0$ a.e. in $\mathbb{R}^{n}$. Let $W_{p}^{k}(w)=W_{p}^{k}\left(\mathbb{R}^{n}, w\right)$ be the weighted Sobolev space, i.e. the space of all functions $f$, which together with their generalized derivatives $D^{\alpha} f$ up to the order $k$ belong to

$$
L_{p}(w)=L_{p}\left(\mathbb{R}^{n} ; w\right)=\left\{f:\|f\|_{L_{p}(w)}^{p}=\int_{\mathbb{R}^{n}}|f(x)|^{p} w(x) d x<\infty\right\},
$$

with the norm

$$
\left\|f\left|W_{p}^{k}(w)\left\|=\sum_{|\alpha| \leq k}\right\| D^{\alpha} f\right| L_{p}(w)\right\|
$$

Only special weights (of type $\left(1+|x|^{2}\right)^{r / 2}$ and their generalizations) and rather sophisticated methods permit to conclude that a function $f$ belongs to $W_{p}^{k}(w)$ if and only if $f w^{1 / p} \in W_{p}^{k}\left(\chi_{\Omega}\right)=W_{p}^{k}$, see [25] and [7] for the so called $W^{n}$ classes (one has to assume that the weighted space in question can be extended to the whole of $\mathbb{R}^{n}$, too). In particular, the class $W^{n}$ excludes singularities so that another approach must be used for weights vanishing or blowing-up at the boundary. The situation is now well understood for weights, which equal to a power of the distance to the boundary. (Note also that such weights can be used to characterize zero traces, even in case of a quite general boundary; see e.g. [14].) The trace theorem for such weights was proved by Nikol'skii in [22] with help of real analysis methods. Let us recall Nikol'skii's result. Assume that $\Omega$ is a domain with a sufficiently smooth boundary $\Gamma$ (as to the required 
smoothness we refer to [22] for details) and let

$$
\varrho(x)=\operatorname{dist}(x, \Gamma), \quad x \in \Omega .
$$

For $k \in \mathbb{N}, 1 \leq p \leq \infty$, and $\gamma \in \mathbb{R}$, denote by $W_{p, \gamma}^{k}$ the weighted Sobolev space with the norm

$$
\left\|f \mid W_{p, \gamma}^{k}\right\|=\|f\|_{L_{p}(\Omega)}+\sum_{|\alpha|=k}\left\|\left(D^{\alpha} f\right) \varrho^{-\gamma}\right\|_{L_{p}(\Omega)} .
$$

Suppose that

$$
0<k+\gamma-1 / p<k
$$

Then

$$
W_{p, \gamma}^{k}(\Omega) \hookrightarrow W_{p}^{k+\gamma-1 / p}(\Gamma)
$$

and, moreover, there exists a bounded extension operator

$$
\operatorname{ext}_{\gamma}: W_{p}^{k+\gamma-1 / p}(\Gamma) \rightarrow W_{p, \gamma}^{k}(\Omega)
$$

A by far more general setting - spaces on fractals with this type of weights - was recently considered by Piotrowska in [23].

In the following we shall make use of a Fourier analytic approach to Sobolev spaces and their weighted generalizations, therefore we recall the most important definitions and fix the notation.

Let $\left\{\varphi_{j}\right\}_{j=0}^{\infty}$ be the smooth (dyadic) decomposition of unity (see [29], [5]): $\operatorname{supp} \varphi_{j} \subseteq\left\{2^{j-1} \leq|\xi| \leq 2^{j+1}\right\}$ for $j \in \mathbb{N}_{0}$ and $\operatorname{supp} \varphi_{0} \subseteq \overline{B_{1}(0)}$ and $\varphi_{j}(\xi)=\varphi_{1}\left(2^{-j+1} \xi\right)$ for $j \in \mathbb{N}$.

For $1 \leq p \leq \infty, 1 \leq q \leq \infty$ and $s \in \mathbb{R}^{1}$ the Besov space $B_{p q}^{s}=B_{p q}^{s}\left(\mathbb{R}^{n}\right)$ is the space of all $f \in \mathcal{S}^{\prime}\left(\mathbb{R}^{n}\right)$ with the finite norm

$$
\left\|f \mid B_{p q}^{s}\right\|=\left(\sum_{k=0}^{\infty} 2^{k s q}\left\|\mathcal{F}^{-1}\left(\varphi_{k} \widehat{f}\right)\right\|_{L_{p}}^{q}\right)^{1 / q}
$$

if $q<\infty$ and with the finite norm

$$
\left\|f \mid B_{p \infty}^{s}\right\|=\sup _{k} 2^{k s}\left\|\mathcal{F}^{-1}\left(\varphi_{k} \widehat{f}\right)\right\|_{L_{p}}
$$

if $q=\infty$, where $\mathcal{F}^{-1}$ is the inverse Fourier transform and $\widehat{f} \equiv \mathcal{F} f$ denotes the Fourier transform of $f$. Replacing the $L_{p}$ space in the above definitions by $L_{p}(w)$ we get a formal definition of the weighted Besov space $B_{p q}^{s}\left(\mathbb{R}^{n} ; w\right)$. Here $\mathcal{S}\left(\mathbb{R}^{n}\right)$ denotes the space of smooth rapidly decreasing functions $f: \mathbb{R}^{n} \rightarrow \mathbb{C}$ and $\mathcal{S}^{\prime}\left(\mathbb{R}^{n}\right)=\left(\mathcal{S}\left(\mathbb{R}^{n}\right)\right)^{\prime}$ its dual. 
We shall also use the Bessel potential spaces $H_{p}^{s}=H_{p}^{s}\left(\mathbb{R}^{n}\right)$ and their weighted clones: For $s$ real and $1<p<\infty$,

$$
\begin{aligned}
H_{p}^{s}\left(\mathbb{R}^{n}\right) & =\left\{f \in \mathcal{S}^{\prime}\left(\mathbb{R}^{n}\right):\left\|\mathcal{F}^{-1}\left[\left(1+|\xi|^{2}\right)^{s / 2} \widehat{f}\right]\right\|_{L_{p}}<\infty\right\}, \\
H_{p}^{s}\left(\mathbb{R}^{n} ; w\right) & =\left\{f \in \mathcal{S}^{\prime}\left(\mathbb{R}^{n}\right):\left\|\mathcal{F}^{-1}\left[\left(1+|\xi|^{2}\right)^{s / 2} \widehat{f}\right]\right\|_{L_{p}(w)}<\infty\right\}
\end{aligned}
$$

normed in the obvious way.

For Lipschitz domains there exists a universal extension operator working on Sobolev, Besov and Bessel potential spaces (and also on the LizorkinTriebel spaces, even for all real $s$, see Rychkov [24]); this means that many relevant properties of spaces on Lipschitz domains follow from the claims on the whole of $\mathbb{R}^{n}$. That is, one can work either with a formal definition of spaces on domains as factorspaces of spaces on $\mathbb{R}^{n}$ modulo equality on the domain in question or with a space on the domain with a usual intrinsic norm (if it is available). This can be partly extended to weighted spaces with the Muckenhoupt weights. Recall that a weight $w$ belongs to the Muckenhoupt class $A_{p}\left(\mathbb{R}^{n}\right)(1<p<\infty)$ if

$$
\sup _{Q}\left(\frac{1}{|Q|} \int_{Q} w(x) d x\right)\left(\frac{1}{|Q|} \int_{Q} w(x)^{1 /(p-1)} d x\right)^{p-1}<\infty,
$$

where the supremum is taken over all cubes $Q \subset \mathbb{R}^{n}$ with edges parallel to the coordinate axes. We shall write simply $A_{p}$ if no misunderstanding can occur. Note in passing that $x^{\prime}=\left(x_{1}, \ldots, x_{n-1}\right) \mapsto\left|x^{\prime}\right|^{\alpha}$ belongs to $A_{q}$ in $\mathbb{R}^{n-1}$ if and only if $-(n-1)<\alpha<(q-1)(n-1)$ (see e.g. [8]).

We also refer to Chua [6] for an extension theorem for Sobolev spaces on domains and to Rychkov [24] as to the formulae for the norm in Sobolev spaces with $A_{p}$ weights in terms of a weighted Littlewood-Paley decomposition. Specifically, for a positive integer $k, 1<p<\infty$, and $w \in A_{p}$,

$$
\left\|f \mid W_{p}^{k}\left(\mathbb{R}^{n} ; w\right)\right\| \sim\left\|\left(\sum_{k=0}^{\infty} 2^{2 j k}\left|\mathcal{F}^{-1}\left(\varphi_{k} \widehat{f}\right)(x)\right|^{2}\right)^{1 / 2}\right\|_{L_{p}(w)} .
$$

This holds even for a bigger class of the so called local $A_{p}$ weights (see [24]) (one requires the condition (1.3) only for small cubes).

In Section 4 we also make use of weighted Sobolev spaces of negative order. It is well known that for $1<p<\infty$ and $w \in A_{p}$, the dual space of $L_{p}(w)$ is given by $L_{p^{\prime}}\left(w^{\prime}\right)$ where $\frac{1}{p}+\frac{1}{p^{\prime}}=1$ and $w^{\prime}=w^{-\frac{1}{p-1}} \in A_{p^{\prime}}$. 
Accordingly, for a positive integer $k$ we define

$$
W_{q}^{-k}\left(\mathbb{R}^{n} ; w\right):=\left(W_{q^{\prime}}^{k}\left(\mathbb{R}^{n} ; w^{\prime}\right)\right)^{\prime} .
$$

For more details about weighted spaces of negative order we refer to [24].

To avoid technicalities we shall not deal with the case of Lipschitz domains and we will concentrate on the basic case of a Sobolev space on $\mathbb{R}^{n}$ and a trace on the boundary of a half-space $\mathbb{R}_{+}^{n}$.

Our main result is:

Theorem 1.1. Given $1<q<\infty$ let $\alpha \in(-(n-1),(q-1)(n-1))$. Then

$$
\operatorname{tr}_{\mathbb{R}^{n-1}} W_{q}^{1}\left(\mathbb{R}_{+}^{n} ;|x|^{\alpha}\right)=B_{q q}^{1-\frac{1}{q}}\left(\mathbb{R}^{n-1} ;\left|x^{\prime}\right|^{\alpha}\right) .
$$

For the precise definition of the function spaces we refer to Section 2 below.

The structure of the paper is as follows: In Section 2 we prove some preliminary results concerning weighted spaces. Then in Section 3 the proof of the Theorem 1.1 for $\alpha>0$ is given, based on a suitable estimate of the solution operator to a Dirichlet boundary value problem. Finally, in Section 4 the case $\alpha<0$ is proved by a duality argument.

\section{Preliminary results on weighted function spaces}

By Garcia-Cuerva and Rubio de Francia [13], Theorem 3.9. the following weighted version of the Hörmander-Mikhlin multiplier theorem holds.

Theorem 2.1. Let $m \in C^{n}\left(\mathbb{R}^{n} \backslash\{0\}\right)$ fulfill the property

$$
\left|\partial^{\alpha} m(\xi)\right| \leq K|\xi|^{-|\alpha|}, \quad \text { for every } \xi \in \mathbb{R}^{n} \backslash\{0\}, \quad|\alpha|=0,1, \ldots, n,
$$

for some constant $K>0$. Then $T$ defined by

$$
\widehat{T f}=m \widehat{f} \quad \text { for } f \in \mathcal{S}\left(\mathbb{R}^{n}\right)
$$

extends to a continuous operator on $L_{w}^{q}\left(\mathbb{R}^{n}\right)$ for every $q \in(1, \infty)$ and $w \in A_{q}$.

In [13] this theorem is stated for an even larger class of multipliers $m$. The assertion on the operator norm is not mentioned explicitly, but it follows from the same proof.

Moreover, recall that a smooth function $p: \mathbb{R}^{n} \times \mathbb{R}^{n} \rightarrow \mathbb{C}$ is in the pseudodifferential symbol class $S_{1,0}^{m}\left(\mathbb{R}^{n} \times \mathbb{R}^{n}\right), m \in \mathbb{R}$, if and only if for 
every $\alpha, \beta \in \mathbb{N}_{0}^{n}$ there is a constant $C_{\alpha, \beta}$ such that

$$
\left|\partial_{\xi}^{\alpha} \partial_{x}^{\beta} p(x, \xi)\right| \leq C_{\alpha, \beta}\langle\xi\rangle^{m-|\alpha|}
$$

uniformly in $x, \xi \in \mathbb{R}^{n}$, where $\langle\xi\rangle:=\left(1+|\xi|^{2}\right)^{\frac{1}{2}}$. Moreover, $S_{1,0}^{m}\left(\mathbb{R}^{n} \times \mathbb{R}^{n}\right)$ is a Fréchet space e.g. with respect to the semi-norms

$$
|p|_{S_{1,0}^{m}}^{(N)}=\sup _{|\alpha|+|\beta| \leq N} \sup _{x, \xi \in \mathbb{R}^{n}}\left|\partial_{\xi}^{\alpha} \partial_{x}^{\beta} p(x, \xi)\right|\langle\xi\rangle^{-m+|\alpha|},
$$

cf. e.g. $[18,28]$. It is well known that $\langle\xi\rangle^{m} \in S_{1,0}^{m}\left(\mathbb{R}^{n} \times \mathbb{R}^{n}\right)$, i.e., for every $\alpha \in \mathbb{N}_{0}^{n}$

$$
\left|\partial_{\xi}^{\alpha}\langle\xi\rangle^{m}\right| \leq C_{\alpha}\langle\xi\rangle^{m-|\alpha|}
$$

uniformly in $\xi \in \mathbb{R}^{n}$. This can e.g. be proved by using the fact that $f(a, \xi):=|(a, \xi)|^{m},(a, \xi) \in \mathbb{R}^{n+1} \backslash\{0\}$, is a smooth and homogeneous function of degree $m$.

For $p \in S_{1,0}^{m}\left(\mathbb{R}^{n} \times \mathbb{R}^{n}\right)$ the associated pseudodifferential operator is defined by

$$
p\left(x, D_{x}\right) f=\int_{\mathbb{R}^{n}} e^{i x \cdot \xi} p(x, \xi) \widehat{f}(\xi) \frac{d \xi}{(2 \pi)^{n}}, \quad f \in \mathcal{S}\left(\mathbb{R}^{n}\right),
$$

where $\widehat{f}=\mathcal{F}[f](\xi)$ and $D_{x}=\frac{1}{i} \partial_{x}$. Then $p\left(x, D_{x}\right)$ can be extended to a bounded operator on weighted Bessel potential spaces by the following result due to Marschall [20, Theorem 1]:

Theorem 2.2. Let $1<q<\infty, s \in \mathbb{R}, w \in A_{q}$, and let $p \in$ $S_{1,0}^{m}\left(\mathbb{R}^{n} \times \mathbb{R}^{n}\right), m \in \mathbb{R}$. Then $p\left(x, D_{x}\right)$ defined as above extends to a bounded linear operator $p\left(x, D_{x}\right): H_{q}^{s+m}\left(\mathbb{R}^{n} ; w\right) \rightarrow H_{q}^{s}\left(\mathbb{R}^{n} ; w\right)$. Moreover, there exists $N=N(s, m, n, q) \in \mathbb{N}_{0}$ and $C=C(s, m, n, q)>0$ such that

$$
\left\|\left.p\left(x, D_{x}\right)\left|\mathcal{L}\left(H_{q}^{s+m}\left(\mathbb{R}^{n} ; w\right), H_{q}^{s}\left(\mathbb{R}^{n} ; w\right)\right) \| \leq C\right| p\right|_{S_{1,0}^{m}} ^{(N)}\right.
$$

uniformly in $p \in S_{1,0}^{m}\left(\mathbb{R}^{n} \times \mathbb{R}^{n}\right)$.

Proof. The first part follows directly from [20, Theorem 1]. The second part follows easily from the linearity of the mapping $S_{1,0}^{m}\left(\mathbb{R}^{n} \times \mathbb{R}^{n}\right) \ni p \mapsto$ $p\left(x, D_{x}\right) \in \mathcal{L}\left(H_{q}^{s+m}\left(\mathbb{R}^{n} ; w\right), H_{q}^{s}\left(\mathbb{R}^{n} ; w\right)\right)$ and the fact that the mapping is bounded, which can be easily checked by observing that all constants in the proof of [20, Theorem 1] only depend on some semi-norm $|p|_{S_{1,0}^{m}}^{(N)}$ with a sufficiently large $N \in \mathbb{N}_{0}$. 
Let $\omega \in A_{q}\left(\mathbb{R}^{n}\right)$, let $\varphi_{j}, j \in \mathbb{N}_{0}$, be a dyadic decomposition of unity as in the introduction and let $s \in \mathbb{R}, 1 \leq p, q \leq \infty$. Note that $\varphi_{j}, j \in \mathbb{N}_{0}$, can be chosen such that $\varphi_{j}(\xi)=\left(\varphi_{1}\left(2^{-j+1} \xi\right)\right.$ for all $j \geq 1$. In particular, this implies

$$
\left|\partial_{\xi}^{\alpha} \varphi_{j}(\xi)\right| \leq C_{\alpha} 2^{-j|\alpha|}
$$

uniformly in $j \in \mathbb{N}_{0}$ and for all $\alpha \in \mathbb{N}_{0}$.

With the notation as in (2.2) we can define weighted Besov space by

$$
\begin{aligned}
B_{p q}^{s}\left(\mathbb{R}^{n} ; \omega\right) & =\left\{f \in \mathcal{S}^{\prime}\left(\mathbb{R}^{n}\right):\|f\|_{B_{p q}^{s}\left(\mathbb{R}^{n} ; \omega\right)}<\infty\right\}, \\
\|f\|_{B_{p q}^{s}\left(\mathbb{R}^{n} ; \omega\right)} & =\left(\sum_{j=0}^{\infty} 2^{s q j}\left\|\varphi_{j}\left(D_{x}\right) f\right\|_{L^{p}\left(\mathbb{R}^{n} ; \omega\right)}^{q}\right)^{1 / q}
\end{aligned}
$$

with the obvious modifications if $q=\infty$. We note that $B_{p q}^{s}\left(\mathbb{R}^{n} ; \omega\right)$ is a retract of $\ell_{q}^{s}\left(\mathbb{N}_{0} ; L^{p}\left(\mathbb{R}^{n} ; \omega\right)\right)$, where

$$
\begin{aligned}
\ell_{q}^{s}\left(\mathbb{N}_{0} ; X\right) & =\left\{\left(a_{j}\right)_{j \in \mathbb{N}_{0}} \in X^{\mathbb{N}_{0}}:\left\|\left(a_{j}\right)_{j \in \mathbb{N}_{0}}\right\|_{\ell_{q}^{s}\left(\mathbb{N}_{0} ; X\right)}<\infty\right\}, \\
\left\|\left(a_{j}\right)_{j \in \mathbb{N}_{0}}\right\|_{\ell_{q}^{s}\left(\mathbb{N}_{0} ; X\right)} & =\left(\sum_{j=0}^{\infty} 2^{s j q}\left\|a_{k}\right\|_{X}^{q}\right)^{\frac{1}{q}} \quad \text { if } q<\infty, \\
\left\|\left(a_{j}\right)_{j \in \mathbb{N}_{0}}\right\|_{\ell_{\infty}^{s}\left(\mathbb{N}_{0} ; X\right)} & =\sup _{j \in \mathbb{N}_{0}} 2^{s j}\left\|a_{j}\right\|_{X} .
\end{aligned}
$$

More precisely, the retractions and coretractions are given by

$$
\begin{aligned}
R: \ell_{q}^{s}\left(\mathbb{N}_{0} ; L^{p}\left(\mathbb{R}^{n} ; \omega\right)\right) \rightarrow B_{p q}^{s}\left(\mathbb{R}^{n} ; \omega\right), & R\left(\left(a_{j}\right)_{j \in \mathbb{N}_{0}}\right) & =\sum_{j=0}^{\infty} \psi_{j}\left(D_{x}\right) a_{j}, \\
S: B_{p q}^{s}\left(\mathbb{R}^{n} ; \omega\right) \rightarrow \ell_{q}^{s}\left(\mathbb{N}_{0} ; L^{p}\left(\mathbb{R}^{n} ; \omega\right)\right), & S f & =\left(\varphi_{j}\left(D_{x}\right) f\right)_{j \in \mathbb{N}_{0}},
\end{aligned}
$$

where $\psi_{j}(\xi)=\varphi_{j-1}(\xi)+\varphi_{j}(\xi)+\varphi_{j+1}(\xi), j \in \mathbb{N}_{0}$, and $\varphi_{-1}(\xi) \equiv 0$.

\subsection{Interpolation of weighted Besov spaces.}

Lemma 2.3. Let $1<q<\infty, s \in \mathbb{R}$, and let $\omega \in A_{q}\left(\mathbb{R}^{n}\right)$. Then

$$
B_{q 1}^{s}\left(\mathbb{R}^{n} ; \omega\right) \hookrightarrow H_{q}^{s}\left(\mathbb{R}^{n} ; \omega\right) \hookrightarrow B_{q \infty}^{s}\left(\mathbb{R}^{n} ; \omega\right) .
$$

Proof. First of all,

$$
\left|\partial_{\xi}^{\alpha}\left(\langle\xi\rangle^{s} \varphi_{j}(\xi)\right)\right| \leq C_{\alpha, s} 2^{s j}\langle\xi\rangle^{-|\alpha|}
$$


for all $\alpha \in \mathbb{N}_{0}^{n}, s \in \mathbb{R}$, because of (2.1), (2.3), and since $c 2^{j} \leq|\xi| \leq C 2^{j}$ on $\operatorname{supp} \varphi_{j}$. Hence

$$
\left\|\left\langle D_{x}\right\rangle^{s} \varphi_{j}\left(D_{x}\right) f\right\|_{L^{q}\left(\mathbb{R}^{n} ; \omega\right)} \leq C_{s} 2^{s j}\|f\|_{L^{q}\left(\mathbb{R}^{n} ; \omega\right)}
$$

by the Mikhlin multiplier theorem for weighted $L^{q}$-spaces, Theorem 2.1, or Theorem 2.2 with $C_{s}$ independent of $j \in \mathbb{N}_{0}$. Since $\varphi_{j}\left(D_{x}\right) f=$ $\varphi_{j}\left(D_{x}\right)\left(\varphi_{j-1}\left(D_{x}\right) f+\varphi_{j}\left(D_{x}\right) f+\varphi_{j+1}\left(D_{x}\right) f\right)$,

$\left\|\left\langle D_{x}\right\rangle^{s} \varphi_{j}\left(D_{x}\right) f\right\|_{L^{q}\left(\mathbb{R}^{n} ; \omega\right)} \leq C_{s} 2^{s j}\left\|\varphi_{j-1}\left(D_{x}\right) f+\varphi_{j}\left(D_{x}\right) f+\varphi_{j+1}\left(D_{x}\right) f\right\|_{L^{q}\left(\mathbb{R}^{n} ; \omega\right)}$

Therefore

$$
\begin{aligned}
\|f\|_{H_{q}^{s}\left(\mathbb{R}^{n} ; \omega\right)} & \leq \sum_{j=0}^{\infty}\left\|\left\langle D_{x}\right\rangle^{s} \varphi_{j}\left(D_{x}\right) f\right\|_{L^{q}\left(\mathbb{R}^{n} ; \omega\right)} \\
& \leq C \sum_{j=0}^{\infty} 2^{s j}\left\|\varphi_{j-1}\left(D_{x}\right) f+\varphi_{j}\left(D_{x}\right) f+\varphi_{j+1}\left(D_{x}\right) f\right\|_{L^{q}\left(\mathbb{R}^{n} ; \omega\right)} \\
& \leq C\|f\|_{B_{q 1}^{s}\left(\mathbb{R}^{n} ; \omega\right)} .
\end{aligned}
$$

Moreover,

$$
\begin{aligned}
\|f\|_{B_{q \infty}^{s}\left(\mathbb{R}^{n} ; \omega\right)} & =\sup _{j \in \mathbb{N}_{0}} 2^{s j}\left\|\left\langle D_{x}\right\rangle^{-s} \varphi_{j}\left(D_{x}\right)\left\langle D_{x}\right\rangle^{s} f\right\|_{L^{q}\left(\mathbb{R}^{n} ; \omega\right)} \\
& \leq C\left\|\left\langle D_{x}\right\rangle^{s} f\right\|_{L^{q}\left(\mathbb{R}^{n} ; \omega\right)}=C\|f\|_{H_{q}^{s}\left(\mathbb{R}^{n} ; \omega\right)}
\end{aligned}
$$

by (2.4), which finishes the proof.

Lemma 2.4. Let $s_{0}, s_{1} \in \mathbb{R}, s_{0} \neq s_{1}, 1<p<\infty, 1 \leq q, q_{0}, q_{1} \leq \infty$, $\theta \in(0,1)$, and let $s=(1-\theta) s_{0}+\theta s_{1}$. Then

$$
\left(B_{p q_{0}}^{s_{0}}\left(\mathbb{R}^{n} ; \omega\right), B_{p q_{1}}^{s_{1}}\left(\mathbb{R}^{n} ; \omega\right)\right)_{\theta, q}=B_{p q}^{s}\left(\mathbb{R}^{n} ; \omega\right)
$$

for any weight function $\omega \in A_{p}$.

Proof. Use that $B_{p q_{j}}^{s_{j}}\left(\mathbb{R}^{n} ; \omega\right)$ is a retract of $\ell_{q_{j}}^{s_{j}}\left(\mathbb{N}_{0} ; L^{p}\left(\mathbb{R}^{n} ; \omega\right)\right)$ and apply [5, Theorem 5.6.1].

Corollary 2.5. Let $1<q<\infty, s_{0}, s_{1} \in \mathbb{R}, s_{0} \neq s_{1}, \theta \in(0,1)$ and let $s=(1-\theta) s_{0}+\theta s_{1}$ and let $\omega \in A_{q}\left(\mathbb{R}^{n}\right)$. Then

$$
\left(H_{q}^{s_{0}}\left(\mathbb{R}^{n} ; \omega\right), H_{q}^{s_{1}}\left(\mathbb{R}^{n} ; \omega\right)\right)_{\theta, q}=B_{q q}^{s}\left(\mathbb{R}^{n} ; \omega\right) .
$$

Proof. The corollary follows directly from Lemma 2.3 and Lemma 2.4. 
Corollary 2.6. Let $1<q<\infty$ and let $\omega=\omega\left(x^{\prime}\right) \in A_{q}\left(\mathbb{R}^{n-1}\right)$. Then

$$
\operatorname{tr}_{\mathbb{R}^{n-1}} W_{q}^{1}\left(\mathbb{R}_{+}^{n} ; \omega\right)=\left(L^{q}\left(\mathbb{R}^{n-1} ; \omega\right), W_{q}^{1}\left(\mathbb{R}^{n-1} ; \omega\right)\right)_{1-\frac{1}{q}, q}=B_{q q}^{1-\frac{1}{q}}\left(\mathbb{R}^{n-1} ; \omega\right)
$$

Proof. The first equality follows from

$$
W_{q}^{1}\left(\mathbb{R}_{+}^{n} ; \omega\right)=L^{q}\left(\mathbb{R}_{+} ; W_{q}^{1}\left(\mathbb{R}^{n-1} ; \omega\right)\right) \cap W_{q}^{1}\left(\mathbb{R}_{+} ; L^{q}\left(\mathbb{R}^{n-1} ; \omega\right)\right)
$$

and Lions' trace method for real interpolation, cf. [5, Corollary 3.12.3] or apply [3, Chapter III, Corollary 4.10.2]. The second equality follows from the previous corollary and the fact that $W_{q}^{1}\left(\mathbb{R}^{n} ; \omega\right)=H_{q}^{1}\left(\mathbb{R}^{n} ; \omega\right)=\{f \in$ $\left.\mathcal{S}^{\prime}\left(\mathbb{R}^{n}\right):\left\langle D_{x}\right\rangle f \in L^{q}\left(\mathbb{R}^{n} ; \omega\right)\right\}$, cf. Fröhlich [11, Lemma 3.1] or [9].

2.2. An Embedding for $\boldsymbol{L}^{q}\left(\mathbb{R}^{n} ;|x|^{\alpha}\right)$. Let $(M, \mathcal{B}, \mu)$ be a measure space and let $L^{p, \infty}=L^{p, \infty}(M, \mu), 1 \leq p<\infty$, denote the corresponding weak $L^{p}$-space (the Marcinkiewicz space, see e.g. [5, Section 1.3]. For reader's convenience let us recall that the quasi-norm in $L^{p, \infty}$ is given by $\sup _{t>0} t^{1 / p} f^{*}(t)$ or equivalently by $\sup _{\lambda>0} \lambda m(f, \lambda)^{1 / p}$, where $m(f, \lambda)=$ $\mu(\{x \in M:|f(x)|>\lambda\})$ is the distribution function of $f$, and $f^{*}$ is the non-increasing rearrangement of $f$, the right-continuous generalized inverse to $m(f, \lambda)$, that is, $f^{*}(t)=\inf \{t>0: m(f, \lambda) \leq t\}$. Moreover, for $1 \leq p<\infty$ and $1 \leq q<\infty$, the Lorentz space $L^{p, q}=L^{p, q}(M, \mu)$ (see again e.g. [5, Section 1.3]) is the space of all $\mu$-measurable functions $f$ with the finite quasi-norm

$$
\|f\|_{L^{p, q}(M, \mu)}=\left(\int_{0}^{\infty}\left[t^{1 / p} f^{*}(t)\right]^{q} \frac{d t}{t}\right)^{1 / q} .
$$

Lemma 2.7. Let $1 \leq p, p_{1}, p_{2}<\infty$ such that $\frac{1}{p}=\frac{1}{p_{1}}+\frac{1}{p_{2}}$. Then there exists $C>0$ such that

$$
\|f g\|_{L^{p, \infty}(M, \mu)} \leq C\|f\|_{L^{p_{1}, \infty}(M, \mu)}\|g\|_{L^{p_{2}, \infty}(M, \mu)} .
$$

Proof. Since the mapping $(f, g) \mapsto f g$ is bilinear, it is sufficient to consider the case $\|f\|_{L^{p_{1}, \infty}(M, \mu)},\|g\|_{L^{p_{2}, \infty}(M, \mu)} \leq 1$. Let $\alpha=\frac{p_{1}}{p_{2}}$. Then we either have $|f(x)| \geq|g(x)|^{\alpha}$ or $|f(x)|<|g(x)|^{\alpha}$. Hence

$$
\begin{aligned}
\mu(\{x:|f(x) g(x)| \geq \lambda\}) & \leq \mu\left(\left\{x:|f(x)|^{1+\alpha} \geq \lambda\right\}\right)+\mu\left(\left\{|g(x)|^{1+\frac{1}{\alpha}} \geq \lambda\right\}\right) \\
& \leq \lambda^{-\frac{p_{1}}{1+\alpha}}+\lambda^{-\frac{\alpha p_{2}}{1+\alpha}}=2 \lambda^{-p}
\end{aligned}
$$

for every $\lambda>0$, which finishes the proof. 
Corollary 2.8. Let $1<q<\infty$ and let $0 \leq \alpha<(q-1) n$. Then

$$
L^{q}\left(\mathbb{R}^{n} ;|x|^{\alpha}\right) \hookrightarrow L^{r, \infty}\left(\mathbb{R}^{n}\right) \quad \text { where } \frac{1}{r}=\frac{1}{q}+\frac{\alpha}{q n} .
$$

Proof. Let $p=\frac{q n}{\alpha}$. Then $|x|^{-\frac{\alpha}{q}} \in L^{p, \infty}\left(\mathbb{R}^{n}\right)$ and therefore

$$
\|f\|_{L^{r, \infty}\left(\mathbb{R}^{n}\right)} \leq\left\||x|^{-\frac{\alpha}{q}}\right\|_{L^{p, \infty}\left(\mathbb{R}^{n}\right)}\left\||x|^{\frac{\alpha}{q}}\right\|_{L^{q, \infty}\left(\mathbb{R}^{n}\right)} \leq C\|f\|_{L^{q}\left(\mathbb{R}^{n} ;|x|^{\alpha}\right)} .
$$

For the following we denote

$$
\begin{aligned}
B_{p q,(r)}^{s}\left(\mathbb{R}^{n}, \omega\right) & =\left\{f \in \mathcal{S}^{\prime}\left(\mathbb{R}^{n}\right):\left\|f \mid B_{p q,(r)}^{s}\left(\mathbb{R}^{n} ; \omega\right)\right\|<\infty\right\}, \\
\left\|f \mid B_{p q,(r)}^{s}\left(\mathbb{R}^{n} ; \omega\right)\right\| & =\left(\sum_{j=0}^{\infty}\left\|\varphi_{j}\left(D_{x}\right) f \mid L^{p, r}\left(\mathbb{R}^{n} ; \omega\right)\right\|^{q}\right)^{1 / q}
\end{aligned}
$$

with the obvious modification if $q=\infty$, where $1 \leq p, q, r \leq \infty$ and $s \in \mathbb{R}$, cf. [29, Section 2.4.1]. We need the following simple lemma.

Lemma 2.9. Let $s_{0}, s_{1} \in \mathbb{R}, 1 \leq q_{0}, q_{1}, r_{0}, r_{1} \leq \infty, q_{0} \neq q_{1}, \theta \in(0,1)$, and let $s=(1-\theta) s_{0}+\theta s_{1}, \frac{1}{q}=\frac{1-\theta}{q_{0}}+\frac{\theta}{q_{1}}$. Then

$$
\left(B_{q_{0} q_{0},\left(r_{0}\right)}^{s_{0}}\left(\mathbb{R}^{n} ; \omega\right), B_{q_{1} q_{1},\left(r_{1}\right)}^{s_{1}}\left(\mathbb{R}^{n} ; \omega\right)\right)_{\theta, q}=B_{q q}^{s}\left(\mathbb{R}^{n} ; \omega\right) .
$$

Proof. First of all, we note that $B_{q_{j} q_{j},\left(r_{j}\right)}^{s_{j}}\left(\mathbb{R}^{n} ; \omega\right)$ and $B_{q q}^{s}\left(\mathbb{R}^{n} ; \omega\right)$ are retracts of $\ell_{q_{j}}^{s_{j}}\left(\mathbb{N}_{0} ; L^{q_{j}, r_{j}}\left(\mathbb{R}^{n} ; \omega\right)\right), \ell_{q}^{s}\left(\mathbb{N}_{0} ; L^{q}\left(\mathbb{R}^{n} ; \omega\right)\right)$, resp., with respect to the same retraction mappings. Hence the statement follows from

$$
\begin{aligned}
& \left(\ell_{q_{0}}^{s_{0}}\left(\mathbb{N}_{0} ; L^{q_{0}, r_{0}}\left(\mathbb{R}^{n} ; \omega\right)\right), \ell_{q_{1}}^{s_{1}}\left(\mathbb{N}_{0} ; L^{q_{1}, r_{1}}\left(\mathbb{R}^{n} ; \omega\right)\right)\right)_{\theta, q} \\
& \left.\quad=\ell_{q}^{s}\left(\mathbb{N}_{0} ;\left(L^{q_{0}, r_{0}}\left(\mathbb{R}^{n} ; \omega\right), L^{q_{1}, r_{1}}\left(\mathbb{R}^{n} ; \omega\right)\right)\right)_{\theta, q}\right)=\ell_{q}^{s}\left(\mathbb{N}_{0} ; L^{q}\left(\mathbb{R}^{n} ; \omega\right)\right)
\end{aligned}
$$

where we have used [5, Theorem 5.6.2] and [5, Theorem 5.3.1].

The following theorem is a key result for the proof of Theorem 1.1.

Theorem 2.10. Let $s \in \mathbb{R}, 1<q<\infty$, and let $0<\alpha<(q-1) n$. Then

$$
B_{q q}^{s+\frac{\alpha}{q}}\left(\mathbb{R}^{n} ;|x|^{\alpha}\right) \hookrightarrow B_{q q}^{s}\left(\mathbb{R}^{n}\right) \cap H_{q}^{s}\left(\mathbb{R}^{n}\right) .
$$

Proof. By Corollary $2.8 L^{q}\left(\mathbb{R}^{n} ;|x|^{\alpha}\right) \hookrightarrow L^{r, \infty}\left(\mathbb{R}^{n}\right)$ for all $0<\alpha<$ $(q-1) n$ and $\frac{1}{r}=\frac{1}{q}+\frac{\alpha}{q n}$. Using the generalized Marcinkiewicz interpolation theorem, cf. [5, Theorem 5.3.2] for different values of $q$ yields

$$
L^{q, r}\left(\mathbb{R}^{n} ;|x|^{\alpha}\right) \hookrightarrow L^{r}\left(\mathbb{R}^{n}\right) \quad \text { where } \frac{1}{r}=\frac{1}{q}+\frac{\alpha}{q n}
$$


for all $0<\alpha<(q-1) n$. Hence for all $0<\alpha<(q-1) n$ and $\frac{1}{r}=\frac{1}{q}+\frac{\alpha}{q n}$

$$
B_{q q,(r)}^{s+\frac{\alpha}{q}}\left(\mathbb{R}^{n} ;|x|^{\alpha}\right) \hookrightarrow B_{r q}^{s+\frac{\alpha}{q}}\left(\mathbb{R}^{n}\right) \hookrightarrow B_{q q}^{s}\left(\mathbb{R}^{n}\right) \cap H_{q}^{s}\left(\mathbb{R}^{n}\right)
$$

due to [29, Section 2.8.2, Equation (2) and (18)]. Hence using Lemma 2.9 for $B_{q q,(r)}^{s+\frac{\alpha}{q}}\left(\mathbb{R}^{n} ;|x|^{\alpha}\right)$ with different values of $q$ together with

$$
\left(B_{q_{0} q_{0}}^{s}\left(\mathbb{R}^{n}\right), B_{q_{1} q_{1}}^{s}\left(\mathbb{R}^{n}\right)\right)_{\theta, q}=B_{q q}^{s}\left(\mathbb{R}^{n}\right),\left(H_{q_{0}}^{s}\left(\mathbb{R}^{n}\right), H_{q_{1}}^{s}\left(\mathbb{R}^{n}\right)\right)_{\theta, q}=H_{q}^{s}\left(\mathbb{R}^{n}\right),
$$

where $\frac{1}{q}=\frac{1-\theta}{q_{0}}+\frac{\theta}{q_{1}}$, cf. [29, Theorem 2.4.1] and [5, Theorem 6.4.5], we conclude (2.6).

\section{Proof for positive $\alpha$}

If $0<\alpha<(q-1)(n-1)$, then $|x|^{\alpha} \geq\left|x^{\prime}\right|^{\alpha}$ and therefore

$$
\operatorname{tr} W_{q}^{1}\left(\mathbb{R}_{+}^{n} ;|x|^{\alpha}\right) \subseteq \operatorname{tr} W_{q}^{1}\left(\mathbb{R}_{+}^{n} ;\left|x^{\prime}\right|^{\alpha}\right)=B_{q q}^{1-\frac{1}{q}}\left(\mathbb{R}^{n-1} ;\left|x^{\prime}\right|^{\alpha}\right)
$$

by Corollary 2.6. Hence it remains to prove the converse inclusion. To this end we use the following extension operator: We denote by $u=K_{D} a$, $a \in \mathcal{S}\left(\mathbb{R}^{n-1}\right)$, the solution of

$$
\begin{aligned}
(1-\Delta) u=0 & & \text { in } \mathbb{R}_{+}^{n}, \\
\left.u\right|_{\partial \mathbb{R}_{+}^{n}}=a & & \text { on } \mathbb{R}^{n-1} .
\end{aligned}
$$

Using partial Fourier transformation $\tilde{a}\left(\xi^{\prime}\right)=\mathcal{F}_{x^{\prime} \mapsto \xi^{\prime}}[a]\left(\xi^{\prime}\right)$ the solution $u=K_{D} a$ can be easily calculated as

$$
u\left(x^{\prime}, x_{n}\right)=K_{D} a=\mathcal{F}_{\xi^{\prime} \mapsto x^{\prime}}^{-1}\left[e^{-\left\langle\xi^{\prime}\right\rangle x_{n}} \tilde{a}\left(\xi^{\prime}\right)\right], \quad x=\left(x^{\prime}, x_{n}\right) \in \mathbb{R}_{+}^{n} .
$$

Note that $\left\langle\xi^{\prime}\right\rangle=\left(1+\left|\xi^{\prime}\right|^{2}\right)^{\frac{1}{2}}$ as above. It is well known that the symbolkernel $\tilde{k}\left(\xi^{\prime}, x_{n}\right):=e^{-\left\langle\xi^{\prime}\right\rangle x_{n}}$ satisfies the following estimate

$$
\sup _{x_{n} \geq 0}\left|x_{n}^{s} \partial_{x_{n}}^{l} \partial_{\xi^{\prime}}^{\alpha} \tilde{k}\left(\xi^{\prime}, x_{n}\right)\right| \leq C_{\alpha, s, l}\left\langle\xi^{\prime}\right\rangle^{l-s-|\alpha|}
$$

uniformly in $\xi^{\prime} \in \mathbb{R}^{n-1}$ and for all $\alpha \in \mathbb{N}_{0}^{n-1}, s \geq 0, l \in \mathbb{N}_{0}$, see e.g. [1, Lemma 2.9]. Using the latter estimate we show

Lemma 3.1. Let $1<q<\infty$, let $s \geq 0$, and let $\omega \in A_{q}\left(\mathbb{R}^{n-1}\right)$. Then $\left(\begin{array}{c}x_{n}^{s} \nabla K_{D} \\ x_{n}^{s} K_{D}\end{array}\right)$ extends to a bounded operator 


$$
\left(\begin{array}{c}
x_{n}^{s} \nabla K_{D} \\
x_{n}^{s} K_{D}
\end{array}\right): B_{q q}^{1-\frac{1}{q}}\left(\mathbb{R}^{n-1} ; \omega\right) \rightarrow \begin{gathered}
L^{q}\left(\mathbb{R}_{+} ; B_{q q}^{s}\left(\mathbb{R}^{n-1} ; \omega\right) \cap H_{q}^{s}\left(\mathbb{R}^{n-1} ; \omega\right)\right)^{n} \\
\times \\
L^{q}\left(\mathbb{R}_{+} ; B_{q q}^{s}\left(\mathbb{R}^{n-1} ; \omega\right) \cap H_{q}^{s}\left(\mathbb{R}^{n-1} ; \omega\right)\right) .
\end{gathered}
$$

Proof. First of all,

$$
\left(\begin{array}{c}
\nabla K_{D} \\
K_{D}
\end{array}\right) a=\mathcal{F}_{\xi^{\prime} \mapsto x^{\prime}}^{-1}\left[\left(\begin{array}{c}
i \xi^{\prime} \\
-\left\langle\xi^{\prime}\right\rangle \\
1
\end{array}\right) e^{-\left\langle\xi^{\prime}\right\rangle x_{n}} \tilde{a}\left(\xi^{\prime}\right)\right] \equiv \mathcal{F}_{\xi^{\prime} \mapsto x^{\prime}}^{-1}\left[\tilde{k}^{\prime}\left(\xi^{\prime}, x_{n}\right) \tilde{a}\left(\xi^{\prime}\right)\right] .
$$

Here $\tilde{k}^{\prime}\left(\xi^{\prime}, x_{n}\right)$ satisfies

$$
\left|\partial_{\xi^{\prime}}^{\alpha} \tilde{k}^{\prime}\left(\xi^{\prime}, x_{n}\right)\right| \leq C_{\alpha, s, l}\left\langle\xi^{\prime}\right\rangle^{1-s-|\alpha|}\left|x_{n}\right|^{-s}
$$

uniformly in $\xi^{\prime} \in \mathbb{R}^{n-1}, x_{n}>0$, and for all $\alpha \in \mathbb{N}_{0}^{n-1}, s \geq 0, l \in \mathbb{N}_{0}$, by virtue of (3.2), (2.1), and the product rule. Hence for every $x_{n}>0$ $\tilde{k}^{\prime}\left(\xi^{\prime}, x_{n}\right) \in S_{1,0}^{1-s}\left(\mathbb{R}^{n-1} \times \mathbb{R}^{n-1}\right)$ is a pseudodifferential symbol with seminorms bounded by $C\left|x_{n}\right|^{-s}$. Thus

$$
\left\|\nabla K_{D} a\left(., x_{n}\right)\right\|_{H_{q}^{s-\frac{1}{p}}\left(\mathbb{R}^{n-1} ; \omega\right)} \leq C\left|x_{n}\right|^{-s}\|a\|_{H_{q}^{1-\frac{1}{p}}\left(\mathbb{R}^{n-1} ; \omega\right)}
$$

by Theorem 2.2. Replacing $s$ by $s+\frac{1}{p}$ we conclude

$$
\left\|x_{n}^{s} \nabla K_{D} a\left(., x_{n}\right)\right\|_{H_{q}^{s}\left(\mathbb{R}^{n-1} ; \omega\right)} \leq C\left|x_{n}\right|^{-\frac{1}{p}}\|a\|_{H_{q}^{1-\frac{1}{p}}\left(\mathbb{R}^{n-1} ; \omega\right)} .
$$

Since $\|f\|_{L^{p, \infty}\left(\mathbb{R}_{+}\right)} \leq\left\|t^{-\frac{1}{p}}\right\|_{L^{p, \infty}}\|f\|_{L^{\infty}} \leq C \sup _{t>0} t^{\frac{1}{p}}|f(t)|$, we get

$$
\left\|x_{n}^{s} \nabla K_{D} a\left(., x_{n}\right)\right\|_{L^{p, \infty}\left(\mathbb{R}_{+} ; H_{q}^{s}\left(\mathbb{R}^{n-1} ; \omega\right)\right)} \leq C\|a\|_{H_{q}^{1-\frac{1}{p}}\left(\mathbb{R}^{n-1} ; \omega\right)} .
$$

Using real interpolation for different values of $p$ and setting $p=q$ afterwards, we conclude

$$
x_{n}^{s} \nabla K_{D}: B_{q q}^{1-\frac{1}{q}}\left(\mathbb{R}^{n-1} ; \omega\right) \rightarrow L^{q}\left(\mathbb{R}_{+} ; H_{q}^{s}\left(\mathbb{R}^{n-1} ; \omega\right)\right)^{n},
$$

where we have used Corollary 2.5, [29, Section 1.18.6, Theorem 2], and (2.8). One more real interpolation with different values of $q$ yields

$$
x_{n}^{s} \nabla K_{D}: B_{q q}^{1-\frac{1}{q}}\left(\mathbb{R}^{n-1} ; \omega\right) \rightarrow L^{q}\left(\mathbb{R}_{+} ; B_{q q}^{s}\left(\mathbb{R}^{n-1} ; \omega\right)\right)^{n},
$$

which completes the proof for $\nabla K_{D}$. The proof for $K_{D}$ is the same. 
Proof of Theorem 1.1, case $\alpha>0$. Using Lemma 3.1 with $s=0$, we conclude $K_{D}: B_{q q}^{1-\frac{1}{q}}\left(\mathbb{R}^{n-1},\left|x^{\prime}\right|^{\alpha}\right) \rightarrow W_{q}^{1}\left(\mathbb{R}_{+}^{n} ;\left|x^{\prime}\right|^{\alpha}\right)$. Moreover, applying Lemma 3.1 with $s=\frac{\alpha}{q}$, we conclude

$\left.x_{n}^{\frac{\alpha}{q}}\left(\begin{array}{c}\nabla K_{D} \\ K_{D}\end{array}\right): B_{q q}^{1-\frac{1}{q}}\left(\mathbb{R}^{n-1},\left|x^{\prime}\right|^{\alpha}\right) \rightarrow L^{q}\left(\mathbb{R}_{+} ; B_{q q}^{\frac{\alpha}{q}}\left(\mathbb{R}^{n-1} ;\left|x^{\prime}\right|^{\alpha}\right)\right)\right) \hookrightarrow L^{q}\left(\mathbb{R}_{+}^{n}\right)$,

where we have also used Theorem 2.10. Hence $K_{D}: B_{q q}^{1-\frac{1}{q}}\left(\mathbb{R}^{n-1},\left|x^{\prime}\right|^{\alpha}\right) \rightarrow$ $W_{q}^{1}\left(\mathbb{R}_{+}^{n} ; x_{n}^{\alpha}\right)$. Since $|x|^{\alpha} \leq C_{\alpha}\left(\left|x^{\prime}\right|^{\alpha}+x_{n}^{\alpha}\right)$, the result for $\alpha>0$ follows.

\section{The result for negative $\alpha$}

The case $\alpha<0$ is derived from the case $\alpha \geq 0$ by a duality argument. More precisely, we use the following abstract lemma.

Lemma 4.1. Let $\omega_{1}, \omega_{2} \in A_{q}$ be given such that one has $\operatorname{tr}_{\mathbb{R}^{n-1}} W_{q}^{1}\left(\mathbb{R}_{+}^{n} ; \omega_{1}\right)=\operatorname{tr}_{\mathbb{R}^{n-1}} W_{q}^{1}\left(\mathbb{R}_{+}^{n} ; \omega_{2}\right)$ with equivalent norms. Then

$$
\operatorname{tr}_{\mathbb{R}^{n-1}} W_{q^{\prime}}^{1}\left(\mathbb{R}_{+}^{n} ; \omega_{1}^{\prime}\right)=\operatorname{tr}_{\mathbb{R}^{n-1}} W_{q^{\prime}}^{1}\left(\mathbb{R}_{+}^{n} ; \omega_{2}^{\prime}\right)
$$

with equivalent norms, where $\frac{1}{q}+\frac{1}{q^{\prime}}$ and $\omega_{j}^{\prime}=\omega_{j}^{-\frac{1}{q-1}}$.

Proof. Let $g \in\left(\operatorname{tr}_{\mathbb{R}^{n-1}} W_{q^{\prime}}^{1}\left(\mathbb{R}_{+}^{n} ; \omega_{1}^{\prime}\right)\right)^{\prime}$ then

$$
G:=\left[\phi \mapsto\langle g, \phi\rangle_{\mathbb{R}^{n-1}}\right] \in\left(W_{q^{\prime}}^{1}\left(\mathbb{R}^{n} ; \omega_{1}^{\prime}\right)\right)^{\prime}=W_{q}^{-1}\left(\mathbb{R}^{n} ; \omega_{1}\right)
$$

with $\|g\|_{\left(\operatorname{tr}_{\mathbb{R}^{n-1}} W_{q^{\prime}}^{1}\left(\mathbb{R}_{+}^{n} ; \omega_{1}^{\prime}\right)\right)^{\prime}}=\|G\|_{W_{q}^{-1}\left(\mathbb{R}^{n} ; \omega_{1}\right)}$.

By [9] one has that $(1-\Delta): W_{q}^{1}\left(\mathbb{R}^{n} ; \omega_{j}\right) \rightarrow W_{q}^{-1}\left(\mathbb{R}^{n} ; \omega_{j}\right), j=1,2$, is an isomorphism. This is a consequence of the Multiplier Theorem 2.1. Indeed, the space $W_{q^{\prime}}^{1}\left(\mathbb{R}^{n} ; \omega_{j}^{\prime}\right)$ can be considered as a closed subspace of $L^{q^{\prime}}\left(\mathbb{R}^{n} ; \omega_{j}^{\prime}\right)^{n+1}$ via the embedding $\phi \mapsto\left(\phi, \frac{\partial}{\partial x_{1}} \phi, \ldots, \frac{\partial}{\partial x_{n}} \phi\right)$. Using this, the Hahn-Banach Theorem and the fact that $L^{q}\left(\mathbb{R}^{n} ; \omega_{j}\right)=\left(L^{q^{\prime}}\left(\mathbb{R}^{n} ; \omega_{j}^{\prime}\right)\right)^{\prime}$ one can show that every $f \in W_{q}^{-1}\left(\mathbb{R}^{n} ; \omega_{j}\right)$ can be represented as $f(\phi)=$ $\left(F_{0}, \phi\right)+\sum_{k=1}^{n}\left(F_{k}, \partial_{k} \phi\right)$ for every $\phi \in C_{0}^{\infty}\left(\mathbb{R}^{n}\right)$ with $F_{k} \in L^{q}\left(\mathbb{R}^{n} ; \omega_{j}\right)$, $k=0, \ldots, n$. Thus

$$
u=\mathcal{F}^{-1}\left(1+|\xi|^{2}\right)^{-1} \mathcal{F} F_{0}+\sum_{k=1}^{n} \mathcal{F}^{-1}\left(1+|\xi|^{2}\right)^{-1} \mathcal{F} \partial_{k} F_{k}
$$

is the unique solution to the equation $(1-\Delta) u=f$ in $\mathbb{R}^{n}$. By Theorem 2.1 it fulfills the estimate $\|u\|_{W_{q}^{1}\left(\mathbb{R}^{n} ; \omega_{j}\right)} \leq c\|f\|_{W_{q}^{-1}\left(\mathbb{R}^{n} ; \omega_{j}\right)}$. 
Since $G$ has its support in $\mathbb{R}^{n-1}$, it follows that $(1-\Delta)^{-1} G$ is a weak solution to the boundary value problem

$$
(1-\Delta)(1-\Delta)^{-1} G=0 \text { on } \Omega
$$

and

$$
(1-\Delta)^{-1} G \in \operatorname{tr}_{\mathbb{R}^{n-1}} W_{q}^{1}\left(\mathbb{R}_{+}^{n} ; \omega_{1}\right),
$$

where $\Omega=\mathbb{R}_{+}^{n}$ or $\Omega=\mathbb{R}_{-}^{n}$. By the a priori estimate of this boundary value problem in [10] one has

$$
\left\|(1-\Delta)^{-1} G\right\|_{W_{q}^{1}\left(\mathbb{R}^{n} ; \omega_{1}\right)} \leq c\left\|\operatorname{tr}_{\mathbb{R}^{n-1}}(1-\Delta)^{-1} G\right\|_{\operatorname{tr}_{\mathbb{R}^{n-1}} W_{q}^{1}\left(\mathbb{R}_{+}^{n} ; \omega_{1}\right)} .
$$

Thus we may estimate using the assumption (4.1)

$$
\begin{aligned}
\|g\|_{\left(\operatorname{tr}_{\mathbb{R}^{n-1}} W_{q^{\prime}}^{1}\left(\mathbb{R}_{+}^{n} ; \omega_{1}^{\prime}\right)\right)^{\prime}} & =\|G\|_{W_{q}^{-1}\left(\mathbb{R}^{n} ; \omega_{1}\right)} \\
& \leq c\left\|(1-\Delta)^{-1} G\right\|_{W_{q}^{1}\left(\mathbb{R}^{n} ; \omega_{1}\right)} \\
& \leq c\left\|\operatorname{tr}_{\mathbb{R}^{n-1}}(1-\Delta)^{-1} G\right\|_{\operatorname{tr}_{\mathbb{R}^{n-1}} W_{q}^{1}\left(\mathbb{R}^{n} ; \omega_{1}\right)} \\
& \leq c\left\|\operatorname{tr}_{\mathbb{R}^{n-1}}(1-\Delta)^{-1} G\right\|_{\operatorname{tr}_{\mathbb{R}^{n-1}} W_{q}^{1}\left(\mathbb{R}^{n} ; \omega_{2}\right)} \\
& \leq c\left\|(1-\Delta)^{-1} G\right\|_{W_{q}^{1}\left(\mathbb{R}^{n} ; \omega_{2}\right)} \\
& \leq c\|G\|_{W_{q}^{-1}\left(\mathbb{R}^{n} ; \omega_{2}\right)} \\
& =c\|g\|_{\left(\operatorname{tr}_{\mathbb{R}^{n-1}} W_{q^{\prime}}^{1}\left(\mathbb{R}_{+}^{n} ; \omega_{2}^{\prime}\right)\right)^{\prime}} .
\end{aligned}
$$

Interchanging the roles of $\omega_{1}$ and $\omega_{2}$, we obtain the reverse estimate

$$
\|g\|_{\left(\operatorname{tr}_{\mathbb{R}^{n-1}} W_{q^{\prime}}^{1}\left(\mathbb{R}_{+}^{n} ; \omega_{2}^{\prime}\right)\right)^{\prime}} \leq c\|g\|_{\left(\operatorname{tr}_{\mathbb{R}^{n-1}} W_{q^{\prime}}^{1}\left(\mathbb{R}_{+}^{n} ; \omega_{1}^{\prime}\right)\right)^{\prime}}
$$

for some $c>0$. We have shown that

$$
\left(\operatorname{tr}_{\mathbb{R}^{n-1}} W_{q^{\prime}}^{1}\left(\mathbb{R}_{+}^{n} ; \omega_{2}^{\prime}\right)\right)^{\prime}=\left(\operatorname{tr}_{\mathbb{R}^{n-1}} W_{q^{\prime}}^{1}\left(\mathbb{R}_{+}^{n} ; \omega_{1}^{\prime}\right)\right)^{\prime} .
$$

Thus, since $\operatorname{tr}_{\mathbb{R}^{n-1}} W_{q^{\prime}}^{1}\left(\mathbb{R}_{+}^{n} ; \omega_{j}^{\prime}\right), j=1,2$, is a factor space of the reflexive Banach space $W_{q^{\prime}}^{1}\left(\mathbb{R}_{+}^{n} ; \omega_{2}^{\prime}\right)$ with respect to a closed subspace, it is reflexive and we obtain

$$
\operatorname{tr}_{\mathbb{R}^{n-1}} W_{q^{\prime}}^{1}\left(\mathbb{R}_{+}^{n} ; \omega_{1}^{\prime}\right)=\operatorname{tr}_{\mathbb{R}^{n-1}} W_{q^{\prime}}^{1}\left(\mathbb{R}_{+}^{n} ; \omega_{2}^{\prime}\right)
$$

as asserted.

Corollary 4.2. Let $-(n-1)<\alpha<0$. Then

$$
\operatorname{tr}_{\mathbb{R}^{n-1}} W_{q}^{1}\left(\mathbb{R}_{+}^{n} ;|x|^{\alpha}\right)=B_{q q}^{1-\frac{1}{q}}\left(\mathbb{R}^{n-1} ;\left|x^{\prime}\right|^{\alpha}\right) .
$$


Proof. Let $\beta:=-\frac{\alpha}{q-1}=-\left(q^{\prime}-1\right) \alpha$. Then $0<\beta<(n-1)\left(q^{\prime}-1\right)$. Thus we are in the range of indices that have already been considered and one obtains from the results of Section 3 that

$$
\operatorname{tr}_{\mathbb{R}^{n-1}} W_{q^{\prime}}^{1}\left(\mathbb{R}_{+}^{n} ;|x|^{\beta}\right)=B_{q^{\prime} q^{\prime}}^{1-\frac{1}{q^{\prime}}}\left(\mathbb{R}^{n-1} ;\left|x^{\prime}\right|^{\beta}\right)=\operatorname{tr}_{\mathbb{R}^{n-1}} W_{q^{\prime}}^{1}\left(\mathbb{R}_{+}^{n} ;\left|x^{\prime}\right|^{\beta}\right),
$$

using Corollary 2.6 and $\left|x^{\prime}\right|^{\beta} \in A_{q^{\prime}}\left(\mathbb{R}^{n-1}\right)$. Thus by Lemma 4.1 we obtain

$$
\operatorname{tr}_{\mathbb{R}^{n-1}} W_{q}^{1}\left(\mathbb{R}_{+}^{n} ;|x|^{\alpha}\right)=\operatorname{tr}_{\mathbb{R}^{n-1}} W_{q}^{1}\left(\mathbb{R}_{+}^{n} ;\left|x^{\prime}\right|^{\alpha}\right)=B_{q q}^{1-\frac{1}{q}}\left(\mathbb{R}^{n-1} ;\left|x^{\prime}\right|^{\beta}\right),
$$

where we again applied Corollary 2.6. This finishes the proof of Theorem 1.1.

Acknowledgements. This work has been developed during the first and the third author's stay at the Institute of Mathematics of the Academy of Sciences of the Czech Republic, which has been supported by the Nečas Center for Mathematical Modeling. This support is gratefully acknowledged. The research of the second author was supported by the Institutional Research Plan no. AV0Z10190503, and by the Grant Agency of the Czech Republic no. 201/06/0400.

\section{References}

[1] H. Abels, Generalized Stokes resolvent equations in an infinite layer with mixed boundary conditions, Math. Nachr., 279 (2006), 1-17.

[2] R.A. Adams, Sobolev Spaces, Academic Press, New York, San Francisco, London, 1975.

[3] H. Amann, Linear and Quasilinear Parabolic Problems, Volume 1: Abstract Linear Theory, Birkhäuser, Basel-Boston-Berlin, 1995.

[4] N. Aronszajn, Boundary value of functions with finite Dirichlet integral, Techn. Report 14, University of Kansas, 1955.

[5] J. Bergh and J. Löfström, Interpolation Spaces, Springer-Verlag, BerlinHeidelberg-New York, 1976.

[6] S.K. Chua, Extension theorems on weighted Sobolev space, Indiana Univ. Math., J. 41 (1992), 1027-1076.

[7] D.E. Edmunds and H. Triebel, Function Spaces, Entropy Numbers and Differential Operators, Cambridge Univ. Press, Cambridge, 1996.

[8] R. Farwig and H. Sohr, Weighted $L^{q}$-theory for the Stokes resolvent in exterior domains, J. Math. Soc., Japan, 49 (1997), 251-288. 
[9] A. Fröhlich, Stokes- und Navier-Stokes-Gleichungen in gewichteten Funktionenräumen, $\mathrm{PhD}$ thesis, TU Darmstadt, Shaker Verlag, Aachen, 2001.

[10] A. Fröhlich, The Stokes operator in weighted $L^{q}$-spaces I: Weighted estimates for the Stokes resolvent problem in a half space, J. Math. Fluid Mech., 5 (2003), 166-199.

[11] A. Fröhlich, Solutions of the Navier-Stokes initial value problem in weighted $L^{q}$-spaces, Math. Nachr., 269/270 (2004), 150-166.

[12] E. Gagliardo, Caratterizzazioni delle tracce sulla frontiera relative ad alcune classi di funzioni in $n$ variabili, Rend. Sem. Mat. Univ., Padova 27 (1957), 284-305.

[13] J. Garcia-Cuerva and J.L. Rubio de Francia, Weighted Norm Inequalities and Related Topics, North Holland, Amsterdam, 1985.

[14] T. Kilpeläinen, J. Kinnunen and O. Martio, Sobolev spaces with zero boundary values on metric spaces, Potential Anal., 12 (2000), 233-247.

[15] V. A. Kondrat'ev, Boundary problems for elliptic equations in domains with conical or angular points (English), Trans. Mosc. Math. Soc., 16 (1967), 227-313.

[16] V. A. Kozlov, V. G. Maz'ya and J. Rossmann, Elliptic Boundary Value Problems in Domains with Point Singularities, Math. Surveys and Monographs Vol. 52, AMS, Providence, R.I., 1997.

[17] A. Kufner, O. John and S. Fučík, Function Spaces, Academia, Prague, 1977.

[18] H. Kumano-Go, Pseudo-Differential Operators, MIT Press, Cambridge, Massachusetts, and London, 1974.

[19] J. L. Lions and E. Magenes, Non-homogeneous Boundary Value Problems and Applications (in French), Dunod, Paris, 1968 (English translation Springer, Berlin-Heidelberg-New York, Vol. I and Vol. 2 1972, Vol. III 1973).

[20] J. Marschall, Weighted $L^{p}$-estimates for pseudo-differential operators with non-regular symbols, Z. Anal. Anwendungen 10 (1985), 493-501.

[21] J. Nečas, Les méthodes directes en équations elliptiques, Academia, Prague, 1967.

[22] S.M. Nikolskii, Approximation of functions of several variables and imbedding theorems 2nd edition (Russian), Moskva, Mir, 1977.

[23] I. Piotrowska, Weighted function spaces and traces on fractals, Dr. rer. nat. thesis, Jena, 2006. 
276 On the trace space of a Sobolev space with a radial weight

[24] V.S. Rychkov, Littlewood-Paley theory and function spaces with $A_{p}^{\text {loc }}$ weights, Math. Nachr., 224 (2001), 145-180.

[25] H.-J. Schmeisser and H. Triebel, Topics in Fourier Analysis and Function Spaces, Geest \& Portig, Leipzig, 1987; Wiley, Chichester, 1987.

[26] L. N. Slobodetskii, Generalized Sobolev spaces and their applications to boundary value problems for partial differential equations (Russian), Ucheb. Zap. Leningrad. Goss. Ped. Inst., 197 (1958), 54-112.

[27] E. M. Stein, The characterization of functions arising as potentials,II, Bull. Amer. Math. Soc., 68 (1962), 577-582.

[28] M.E. Taylor, Pseudodifferential Operators and Nonlinear PDE, Birkhäuser, Boston, 1991.

[29] H. Triebel, Interpolation Theory, Function Spaces, Differential Operators, North-Holland, Amsterdam, New York, Oxford, 1978.

Max Planck Institute for Mathematics in Science

Inselstr. 22, 04103 Leipzig

Germany

(E-mail : abels@mis.mpg.de)

Institute of Mathematics of the ASCR

v.v.i., Žitná 25

11567 Prague 1

Czech Republic

(E-mail :krbecm@math.cas.cz)

Department of Mathematics

Darmstadt University of Technology

Schlossgartenstr. 7

64289 Darmstadt

Germany

(E-mail : schumacher@mathematik.tu-darmstadt.de)

(Received : June 2007) 


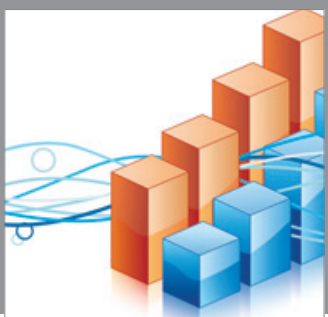

Advances in

Operations Research

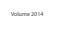

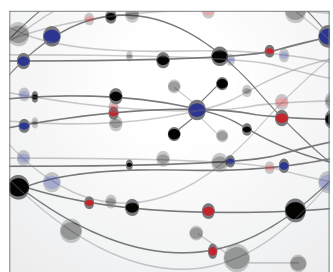

\section{The Scientific} World Journal
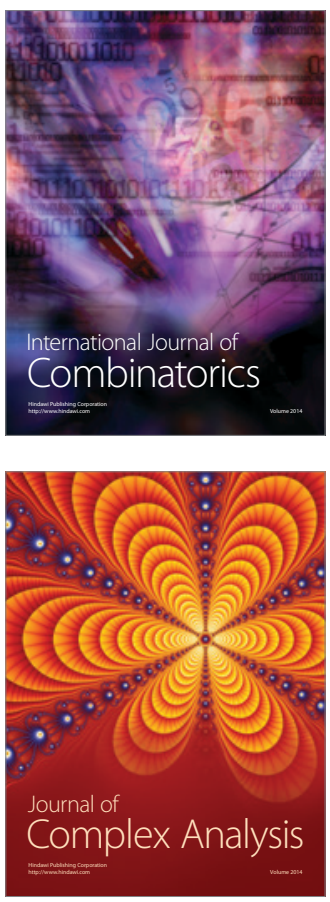

International Journal of

Mathematics and

Mathematical

Sciences
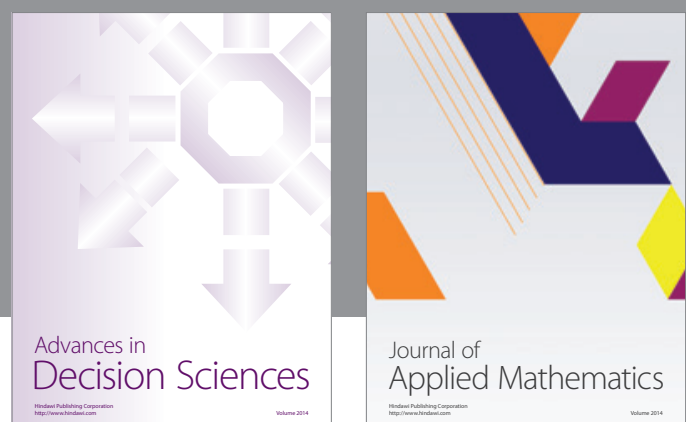

Journal of

Applied Mathematics
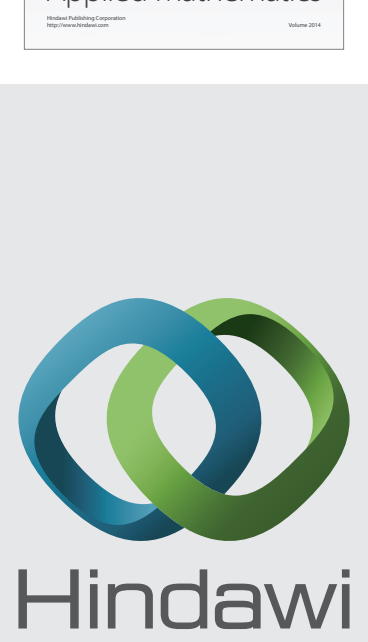

Submit your manuscripts at http://www.hindawi.com
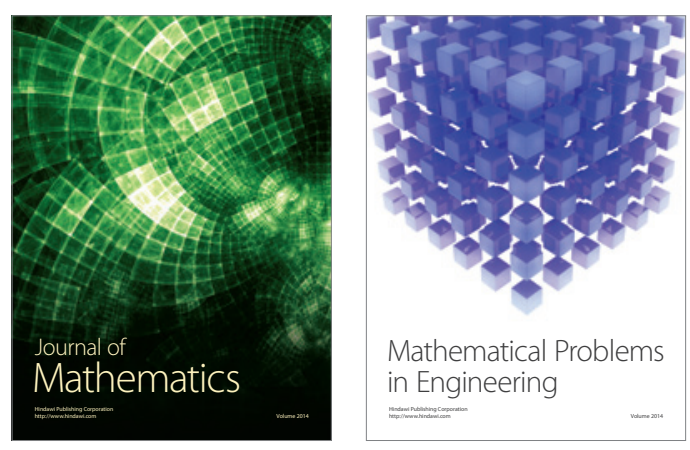

Mathematical Problems in Engineering
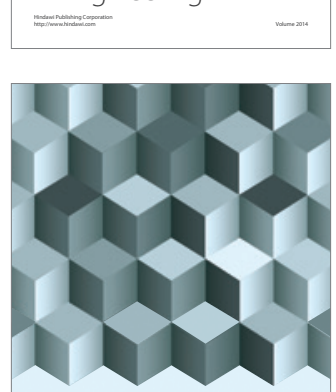

Journal of

Function Spaces
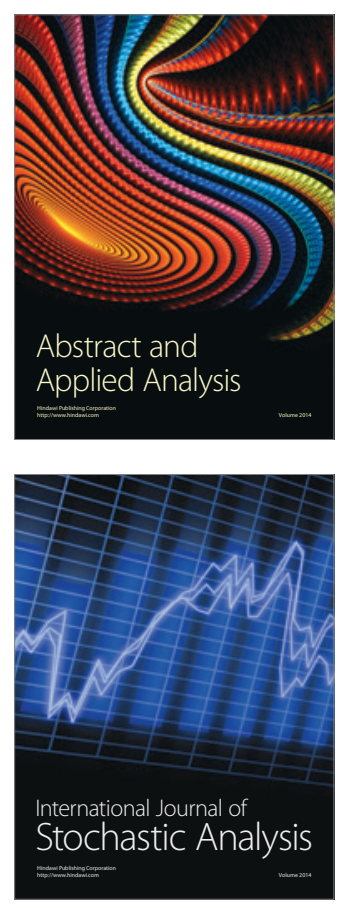

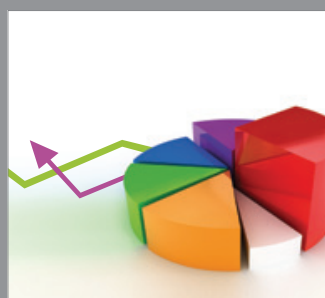

ournal of

Probability and Statistics

Promensencen
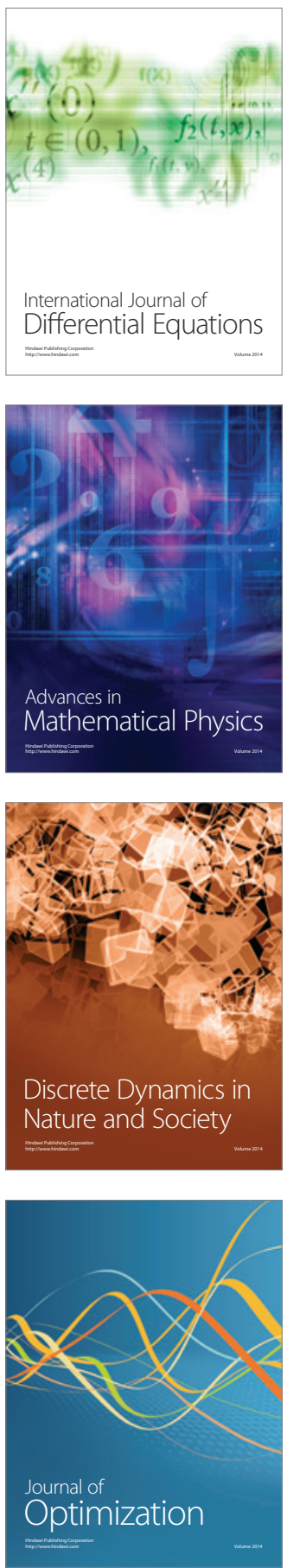